Thorax (1970), 25, 691.

\title{
Necrotizing pulmonary aspergillosis
}

\author{
W. P. U. KENNEDY, D. N. MALONE, and W. B L Y TH \\ Respiratory Diseases Unit, Northern General Hospital, Edinburgh, and Departments of \\ Respiratory Diseases, Medicine, and Botany, University of Edinburgh
}

\begin{abstract}
Necrotizing or invasive aspergillary infection of the lungs has previously been considered a rare condition affecting only the debilitated or seriously ill. Four patients with necrotizing pulmonary aspergillosis are described, and the mycological and histopathological findings are discussed. The diagnosis in the first patient was not made until necropsy, following death from fulminating pulmonary infection. Of three patients treated with natamycin, two made a satisfactory recovery ; the other died later from bronchial carcinoma. Previous multiple antibiotic therapy in each case may have been a contributory factor to infection with Aspergillus fumigatus. We suggest that fungal infection of the lungs should be actively sought in patients with pulmonary disease, especially in those receiving multiple antibiotic or corticosteroid therapy.
\end{abstract}

The most important fungal disease of the lungs in the British Isles at the present time is bronchopulmonary aspergillosis, a condition which is becoming more commonly recognized in a variety of clinical situations. Classifications have been suggested by various authors, such as Gowing and Hamlin (1960) and Campbell and Clayton (1964); here we propose the following simple terminology:

1. Allergic asthma

2. Pulmonary eosinophilia

3. Extrinsic allergic alveolitis

4. Intercavitary aspergilloma

5. Necrotizing pulmonary aspergillosis

6. Disseminated aspergillosis.

This paper describes four adult male patients in whom bronchopulmonary aspergillosis was accompanied by necrosis of lung tissue.

\section{CASE REPORTS}

PATIENT 1 A retired shipping manager aged 74 had been ill for four weeks before admission to hospital with a productive cough, increasing exertional dyspnoea, three episodes of right pleural pain, and a chest radiograph which showed only slight shadowing in the right mid zone. During the first four weeks in hospital, repeated culture of sputum yielded only upper respiratory tract organisms except on one occasion when a moderate growth of a coliform bacillus was obtained together with a scanty growth of Aspergillus fumigatus. Despite treatment with ampicillin, he became steadily worse. The chest radiograph showed progressive bronchopneumonic changes in both lungs, and eventual cavitation in both upper lobes (Fig. 1). A diagnosis of suppurative pneumonia was made, and physiotherapy and treatment with cephaloridine produced a slight improvement in his general condition. About 36 hours before death Pseudomonas aeruginosa or pyocyanea was isolated from the sputum, and antibiotic treatment was altered to gentamycin and erythromycin lactobionate. This did not prevent further deterioration and he died 47 days after admission to hospital. Subsequent mycological examination of the sputum specimens showed numerous colonies of $\boldsymbol{A}$. fumigatus. A specimen of serum obtained after death was examined for precipitating antibodies using extracts of $\boldsymbol{A}$. fumigatus; multiple precipitin lines to all the antigen used were demonstrated.

Necropsy findings The upper lobes of both lungs were lightly adherent to the chest wall by recently formed adhesions and there was a small amount of turbid fluid in both pleural cavities. Both upper lobes were occupied by multiloculated abscess cavities containing shreds of necrotic lung tissue; the abscess walls were lined in some places by a stringy whitish membrane (Fig. 2). Patches of suppurative bronchopneumonia were present in the lower lobes. The trachea and major bronchi contained copious amounts of pus.

Microscopical examination of the membrane lining parts of the larger abscesses revealed a mat of tangled hyphae, which were thick, imperfectly septate, and branched repeatedly in an arborescent pattern. The fungal layer also contained enormous numbers of Gram-negative rods. Other fungal masses, composed of rounded collections of radially orientated hyphae, lay in the pus within bronchioles and in the patches of suppurative bronchopneumonia.

The apical situation of the large abscesses in each lung raised the possibility of colonization of preexisting bronchiectatic spaces, but the necrosis of lung tissue was so extensive that it obscured any evidence 


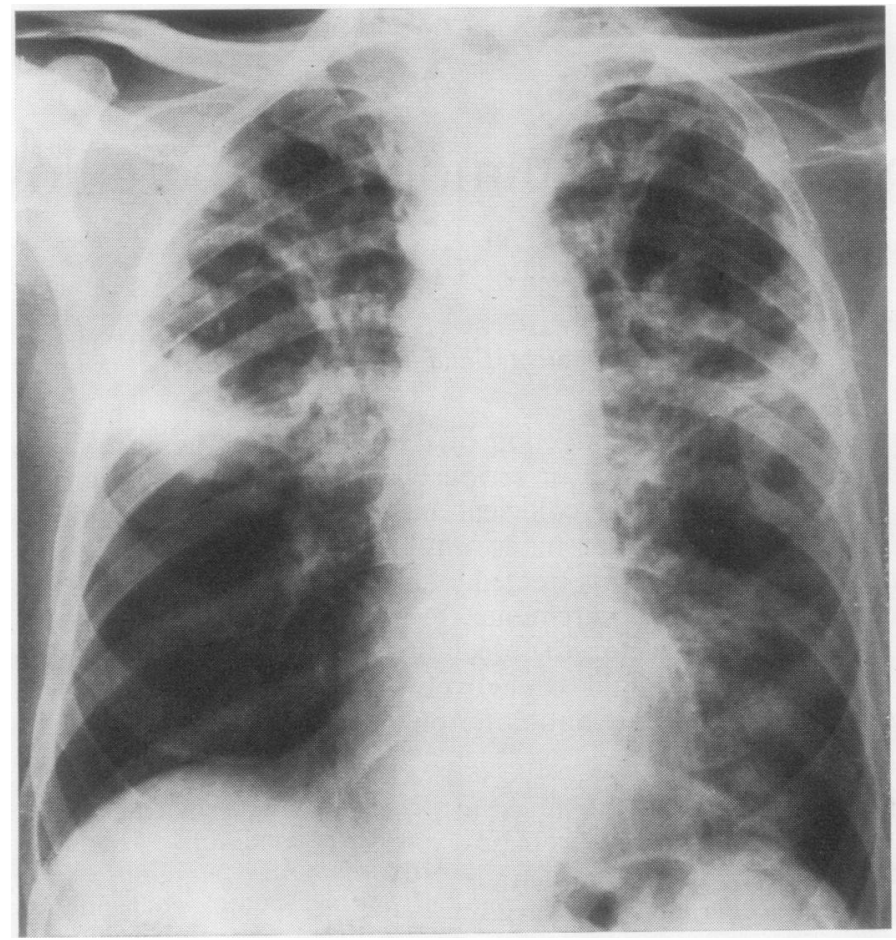

FIG. 1. Patient 1. Chest radiograph shortly before death showing extensive cavitation in both upper lobes.

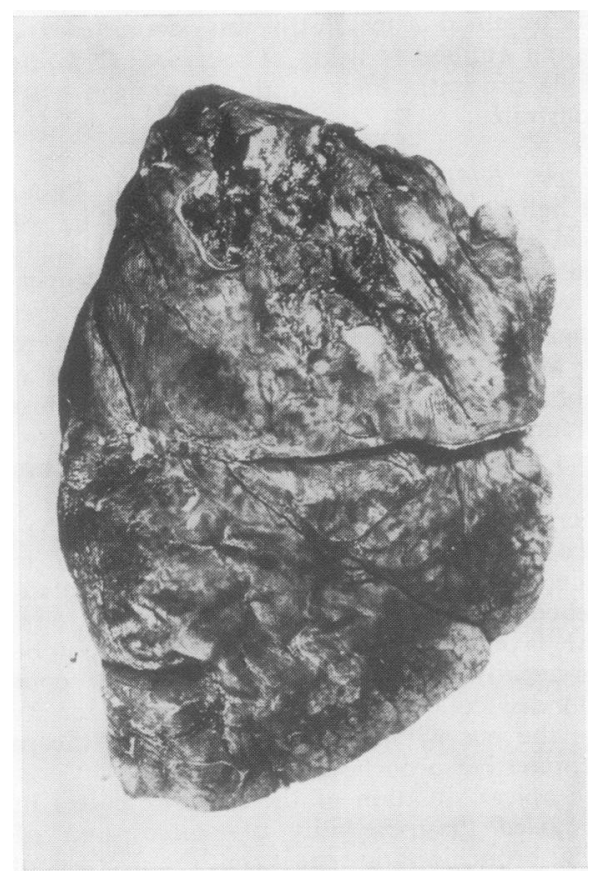

FIG. 2. Patient 1. Lateral view of right lung taken at necropsy. Extensive abscess cavity in upper lobe, lined in places by a whitish membrane of fungus. of previous lung damage which might have predisposed to fungal infection. No evidence of fungal or bacterial infection was found in any organ outside the respiratory system.

PATIENT 2 A 59-year-old baker had a long history of chronic bronchitis. During the four months preceding his acute illness he had received one short course of oxytetracycline for seven days, and two short courses of prednisolone. He was admitted to hospital with acute dyspnoea and a pyrexia of $38.6^{\circ} \mathrm{C}$., but at first the chest radiograph showed only an old calcified tuberculous lesion in the right upper lobe. The sputum, however, was purulent and yielded on culture a pure growth of Streptococcus pneumoniae. He was treated with ampicillin for five days. There was temporary $\mathcal{N}$ improvement, but on the ninth day he was ill once again, with fever and oral thrush. A pneumonia had $O$ developed in the right upper lobe (Fig. 3), and he was $\omega$ expectorating purulent sputum, from which $A$. fumigatus was isolated. A diagnosis of suppurative or 0 necrotizing fungal pneumonia, perhaps secondary to $\frac{C}{\Phi}$ bacterial infection, was made. The detection in the serum of precipitating antibodies against $A$. fumigatus provided supporting evidence for the diagnosis.

An aerosol suspension of natamycin in a dose of $\frac{O}{\mathbb{D}}$ $2.5 \mathrm{mg}$. six-hourly was administered by intermittent positive pressure with a Bennet respirator and con- $\varrho$ tinued for 38 days. By the tenth day of treatment his $\sigma$ temperature was normal, and his clinical condition had improved. The sputum remained purulent, and 0 the upper lobe of the right lung became cavitated 


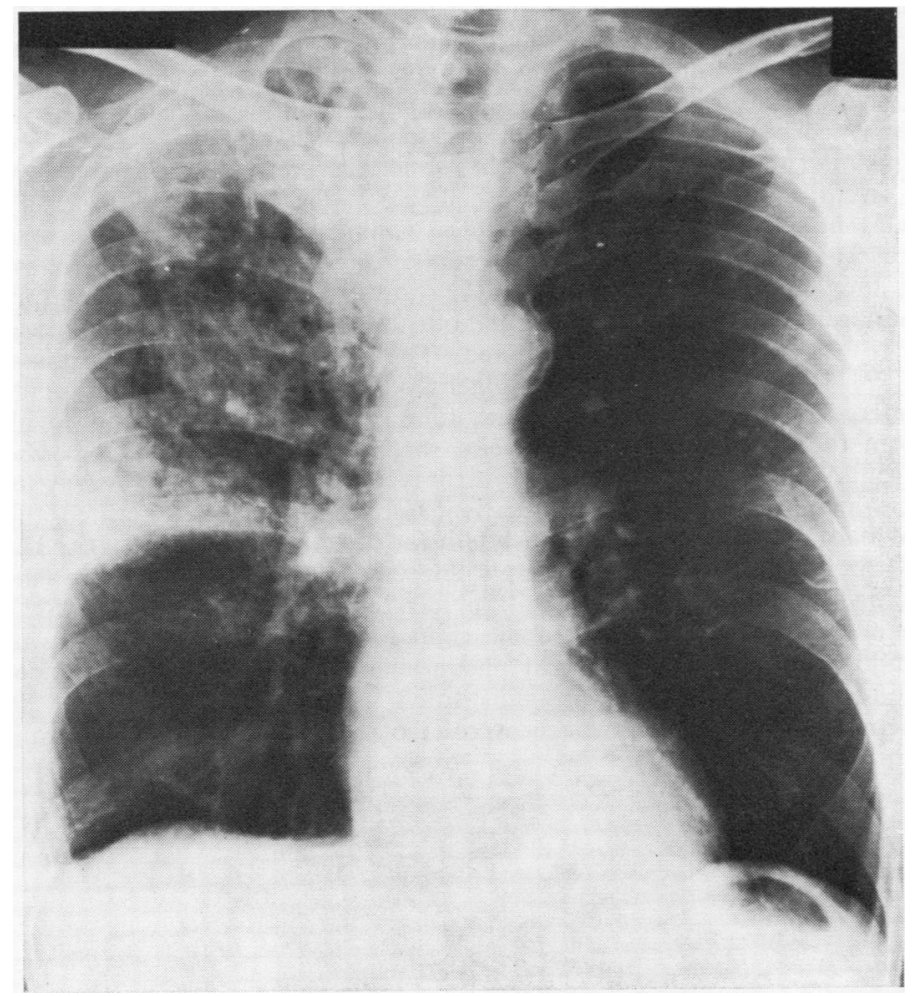

FIG. 3. Patient 2. Chest radiograph showing pneumonic shadowing throughout right upper lobe.

FIG. 4. Patient 2. Tomograph showing cavitation in right upper lobe.

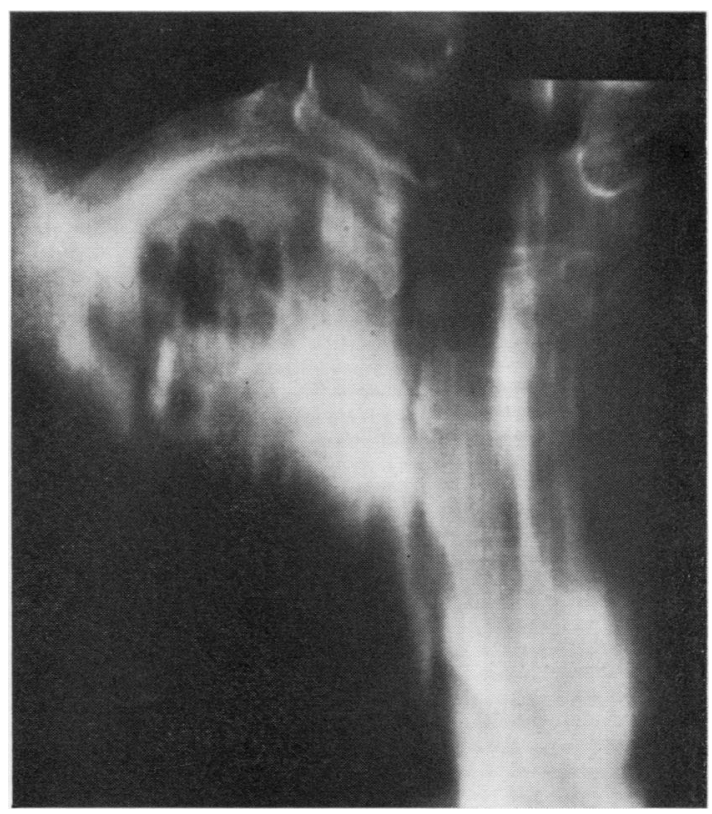


(Fig. 4). By the time that natamycin was discontinued he was feeling well, although the sputum still yielded A. fumigatus on culture. He was discharged home without further antibacterial or antifungal treatment. When seen 10 weeks later his cough had lessened but the sputum was still purulent and $A$. fumigatus was obtained on culture. Serum precipitins were still present. Four months after leaving hospital he was well, with only slight cough, and the sputum was now mucoid. The cavitated lesion in the right lung apex had healed by fibrosis, leaving only a small residual opacity visible in the chest radiograph.

PATIENT 3 A hotel porter aged 48 had suffered from chronic osteomyelitis of the left femur from the age of 13 years. He also had a history of pulmonary tuberculosis with persistent cavitation for which the posterior segment of the right upper lobe had been resected at the age of 36 years. For 10 months before the present illness he had been receiving lincomycin following an episode of acute osteomyelitis associated with septicaemia and subacute bacterial endocarditis. He had now developed a fever, cough productive of copper-coloured sputum, and bilateral pleural pain.
He failed to improve despite treatment with ampicillin for 10 days, and then for five days with trimethoprim and sulphamethoxazole, and he developed severe glossitis. Two days before admission, the left leg became swollen and tender.

He looked thin and ill on admission and was dyspnoeic at rest. The left leg was swollen and discoloured and there was marked calf tenderness. Scattered coarse crepitations were audible in the chest, and the radiograph showed widespread inflammatory shadowing throughout the right lower lobe, which was slightly contracted. The sputum, although purulent, yielded no pathogens on culture. The diagnosis made was of right lower lobe pneumonia or infarction, with left femoral vein thrombosis, and treatment was started with erythromycin and anticoagulants. After one week the patient's condition had deteriorated and he was seriously ill with a pyrexia of $40.0^{\circ} \mathrm{C}$. The chest radiograph showed mottled opacities in the upper part of the left lung field in addition to the shadowing in the right lower lobe. The possibility of staphylococcal pneumonia was considered, and lincomycin was substituted for erythromycin, but no staphylococci were isolated and the serum anti-

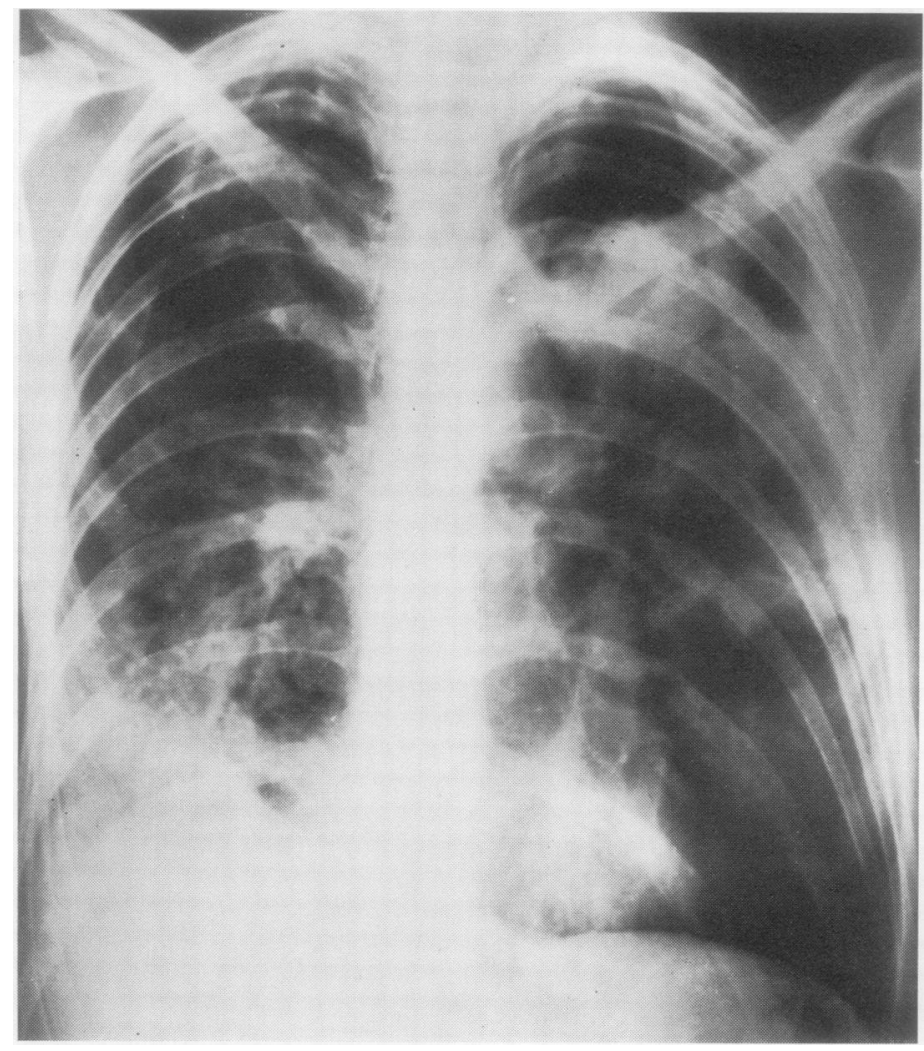

FIG. 5. Patient 3. Chest radiograph showing development of cavitation in left upper lobe. 


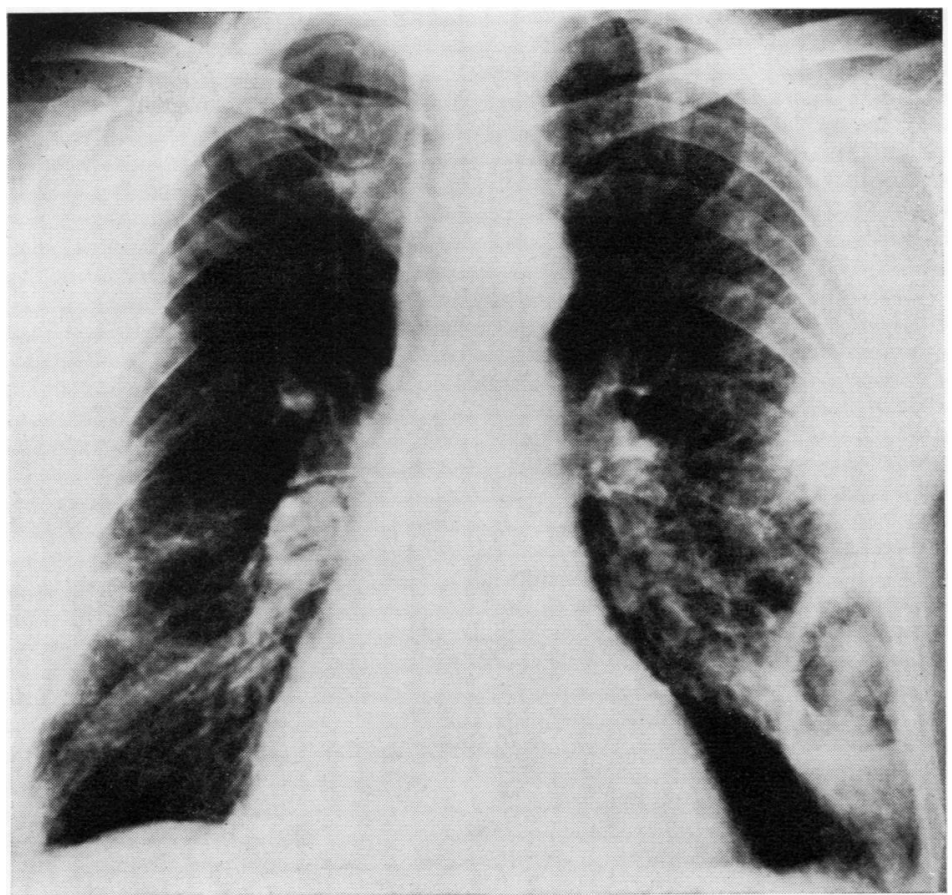

staphylolysin titre was less than 2 units $/ \mathrm{ml}$. At this stage Haemophilus influenzae and $A$. fumigatus were cultured from the sputum, but an aspergillus precipitin test was negative.

As the patient's condition began to improve, the chest radiograph showed some clearing of the right lung field, but cavitation had developed in the left upper lobe, becoming more extensive during the succeeding four weeks (Fig. 5). Serum precipitins against $A$. fumigatus were now demonstrable. Lincomycin was stopped and during the next 15 weeks natamycin was given by inhalation, in doses of $2.5 \mathrm{mg}$. increasing to $5.0 \mathrm{mg}$. thrice daily. $A$. fumigatus in small numbers continued to be isolated from the sputum during the first six weeks of natamycin therapy but not thereafter, nor in the 10 months since treatment was stopped. The aspergillus precipitin test has remained positive. The patient has regained his normal health, and his weight has increased by $13 \mathrm{~kg}$. Chest radiographs show clearing of both lung fields although a small cavity remains at the left apex.

PATIENT 4 A 68-year-old ex-miner, despite having smoked 20 to 25 cigarettes daily during most of his adult life, did not complain of any respiratory symptoms until two weeks before admission to hospital. He then developed fever, left pleural pain, and a cough productive of purulent sputum. The clinical signs were those of bilateral basal bronchopneumonia ; the chest radiograph showed, in addition, a dense opacity in the periphery of the left lower zone. His family doctor had treated him with ampicillin; in hospital he received benzylpenicillin by injection for six days, and when $H$. influenzae was cultured from the sputum, the antibiotic was altered to ampicillin again. During this time the lesion in the left lower lobe enlarged and became cavitated (Fig. 6). Because of its rapid appearance this lesion was thought unlikely to be a tumour, and a diagnosis of lung abscess was made. Chloramphenicol was then given for 10 days. At the end of this time he was feeling better and was afebrile, and the chest radiograph showed that the abscess was smaller, although diffuse inflammatory shadowing was still present in the left lung field. A test for serum precipitins against $A$. fumigatus was negative.

Seven weeks from the onset of the illness he complained of right pleural pain and breathlessness; chest radiographs showed new shadowing in the lower zone of the right lung field. The possibility of a secondary fungal infection was considered, and treatment with ampicillin was given while specific investigations for fungi were carried out. Culture of sputum on the second day yielded a scanty growth of $\boldsymbol{A}$. fumigatus. Serum taken on the same day gave a positive aspergillus precipitin test, but skin testing with aspergillus 


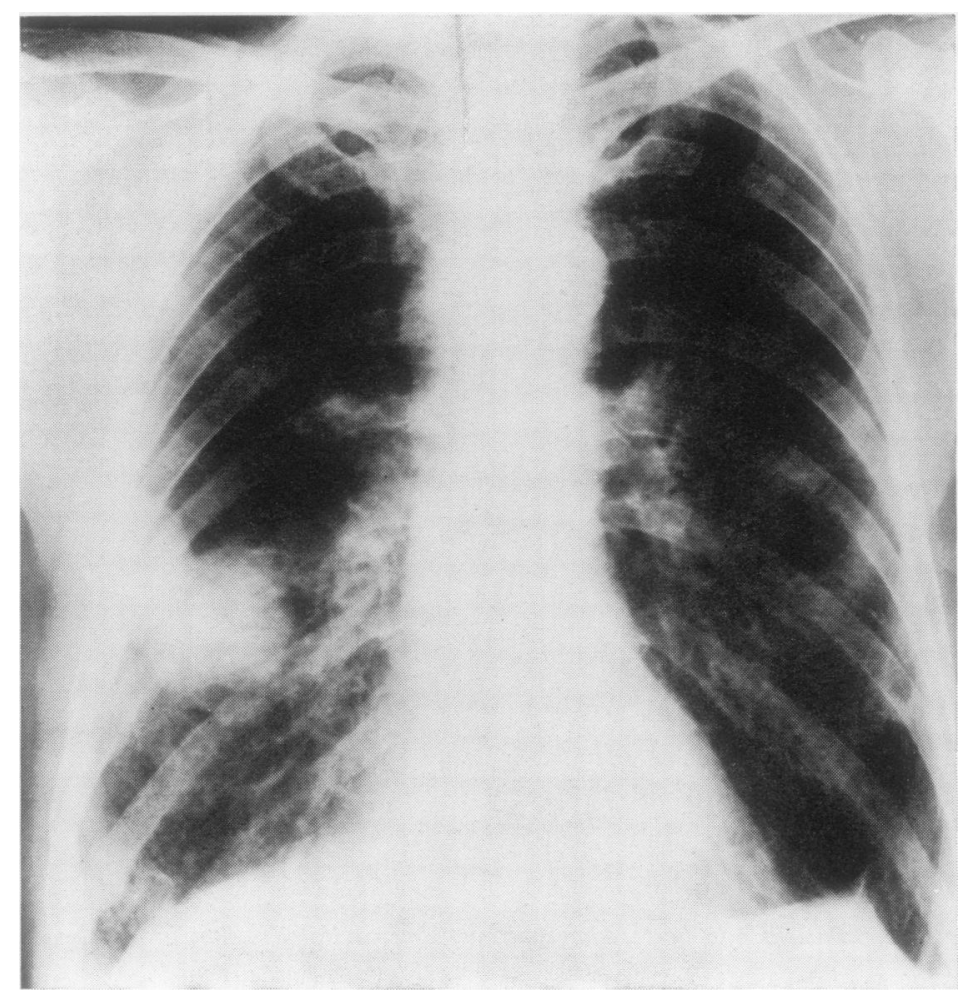

FIG. 7. Patient 4. Chest radiograph taken six months after onset of original illness, showing healing of lesion in left lower lobe, but development of two dense opacities in lower zone of right lung field. The fractures visible in the ninth and tenth right ribs were known to be due to previous trauma.

extract was negative. He was given no further antibiotic, and physiotherapy alone was continued. His general condition gradually improved, his sputum became mucoid, and after three weeks he was discharged home. The chest radiograph at this time showed considerable improvement, but opacity persisted at the site of the original abscess, and there was some residual shadowing in the right lung field just below the transverse fissure.

Four months later it was apparent that the opacity in the right lung field had been slowly enlarging. There were now two dense localized lesions, one within the substance of the subapical segment of the lower lobe, the other situated subpleurally against the lateral chest wall. The left lung field had largely cleared, but there was still a small thin-walled cavity at the site of the abscess in the left lower lobe (Fig. 7). While these radiographic changes were developing he had remained relatively well, although with an intermittent cough productive of mucoid sputum. Silver stains showed that the sputum contained fungal spores of Aspergillus or Penicillium type and of Clado- sporium. Mycological culture yielded profuse growths of a number of Aspergillus species-fumigatus, flavus, restrictus and ochraceus-and Penicillium decumbens. Serum precipitins against aspergillus were still present. While it seemed likely that these lesions were of a similar nature to the original lung abscess, the question of malignant disease had to be considered; a bronchial carcinoma could well have arisen in this elderly man who was a heavy smoker. The patient declined bronchoscopic examination, and it was decided to start antifungal therapy without any further major investigative procedure. He was treated with natamycin inhalations in doses of $2.5 \mathrm{mg}$. thrice daily, and within three weeks mycological culture of the sputum was completely negative. After cessation of treatment $A$. fumigatus appeared once again in the sputum together with several other species of mould.

His condition thereafter deteriorated, and the chest radiograph made it plain that a carcinoma was present, eroding the sixth right rib and producing lymph node enlargement in the right paratracheal region. Palliative radiotherapy relieved the chest wall 
pain, but he died at home, nine months after the onset of his original illness: permission for necropsy was not obtained.

\section{MYCOLOGICAL METHODS}

SPUTUM SMEARS Sputum samples were temporarily stored at $4^{\circ}$ C. prior to removal to slides for direct microscopy. Smears were stained by the Grocott methenamine silver method (Grocott, 1955) and were given a final light wash with Delafield's haematoxylin. This method was considered superior, for primary visualization and differentiation, to both the periodic acid-Schiff technique used by Orie, de Vries, and Kikstra (1960) and the pancreatin-potassium hydroxide method described by English and Henderson (1967).

SPUTUM CULTURE Each sputum sample was divided equally between two Petri dish plates of malt agar; one plate was incubated at $26^{\circ} \mathrm{C}$., the other at $37^{\circ} \mathrm{C}$. All fungi arising on the plates were identified, and their densities were recorded as indicated by colony counts.

LUNG SECTIONS AND CULTURE A portion of lung from patient 1 was examined by staining selected sections by the periodic acid-Schiff and Grocott methenamine silver schedules. After exploratory dissection, selected areas of lung were surface sterilized by heat, and small tissue blocks from both intact and necrotic zones were removed aseptically to malt agarplates for incubation at $26^{\circ} \mathrm{C}$. and $37^{\circ} \mathrm{C}$.

\section{MYCOLOGICAL RESULTS}

SPUTUM SMEARS Smears from patient 4 yielded conidia of Aspergillus or Penicillium type on five occasions, and septate mycelium on two. Their occurrence correlated with that of $A$. fumigatus in culture. This patient also expectorated easily identifiable conidia of Alternaria sp. and Cladosporium sp.

SPUTUM CULTURE All four patients showed numerous colonies of $A$. fumigatus either from single sputum samples or from successive samples. There was also a random background flora which included: Aspergillus spp.-flavus, restrictus, ochraceus, glaucus, niger, terreus; Penicillium spp.frequentans, brevicompactum, commune, digitatum, jenseni, roqueforti; Alternaria sp.; Ulocladium sp.; Botrytis sp.; Trichoderma sp.; Candida spp.

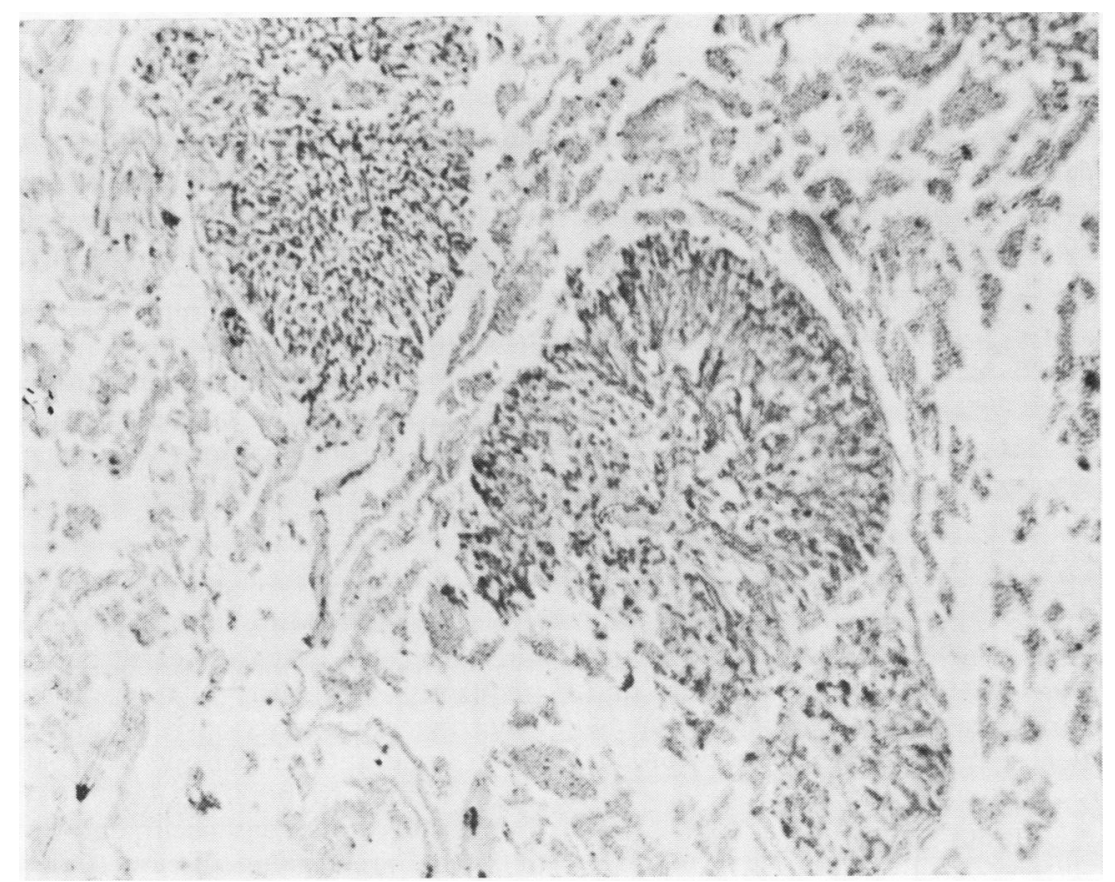

FIG. 8. Patient 1. Mycelial colonies of A. fumigatus in necrotic lung tissue $(\mathrm{PAS} \times 70)$. 


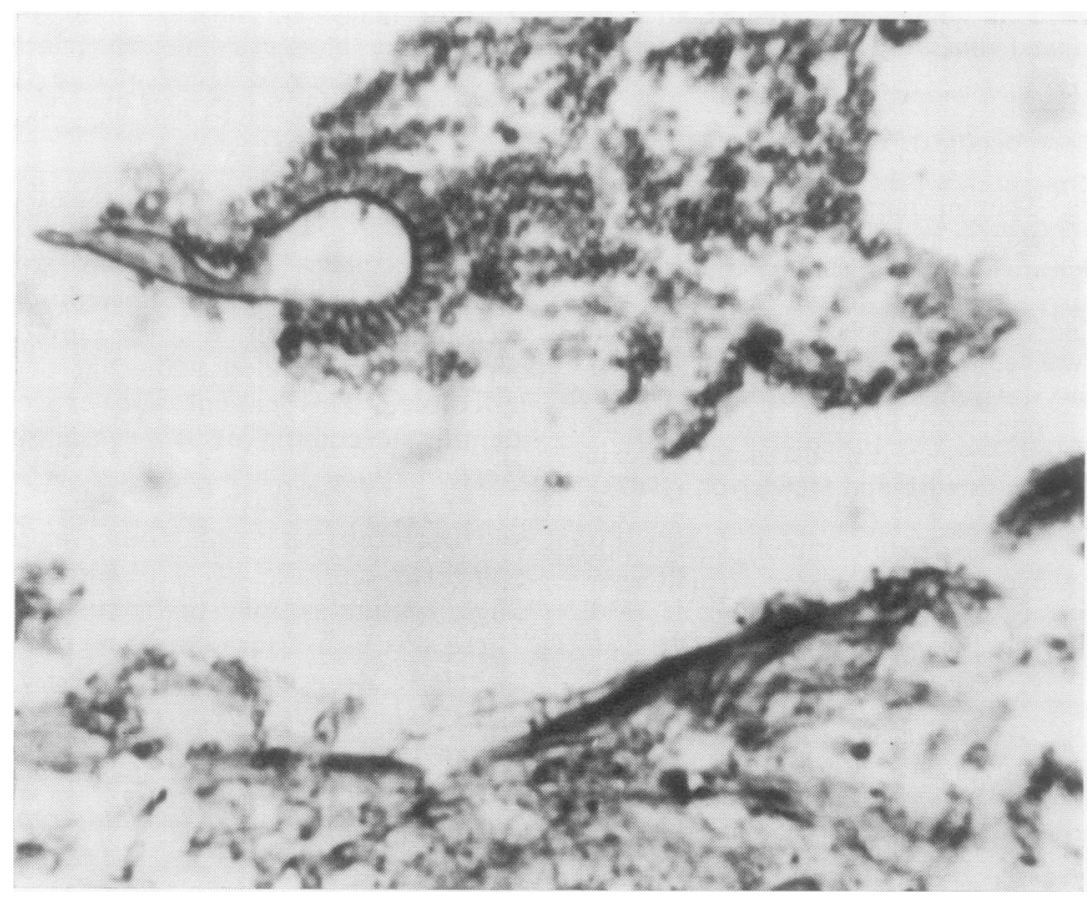

FIG. 9. Patient 1. Lung at necropsy showing intra-alveolar sporulation of A. fumigatus (PAS $\times 600)$.

LUNG HISTOPATHOLOGY AND CULTURE The lung sample from patient 1 contained well-developed mycelial colonies, with intra-alveolar sporulating conidiophores of $\boldsymbol{A}$. fumigatus (Figs 8 and 9). A close association between the hyphae and Pseudomonas organisms was noted. Identifications and colony counts on malt agar showed the presence of Ps. aeruginosa, $A$. fumigatus and Candida albicans.

\section{SEROLOGICAL METHODS}

Serum specimens were examined for precipitin antibody using Ouchterlony plates cast with agar containing citrate to eliminate possible error due to 'C-substance' (Ouchterlony, 1953; Longbottom and Pepys, 1964). The number and intensity of the precipitation lines using extracts of A. fumigatus (Bencard) as antigen were recorded.

\section{DISCUSSION}

This communication describes a destructive and potentially fulminating fungal infection of the lungs, which perhaps occurs more frequently than is commonly appreciated. The fact that aspergilli can cause human pulmonary disease has been known since the middle of the last century, and in their paper of 1952 Hinson, Moon, and Plummer provide an illuminating historical review of human invasion by this fungus. The vast majority of reports since then, however, have been concerned with the saprophytic colonization of abnormal bronchopulmonary tissue to form a 'fungus ball' or aspergilloma; and with the hypersensitive or allergic varieties first recognized by Hinson et al. (1952).

Several reports of the invasive form of pulmonary aspergillosis have appeared in the literature over the past 20 years, but the disease is frequently described as rare or very rare and occurring only in seriously ill or debilitated subjects. Some authors consider the pathogenicity of $A$. fumigatus to be slight, and so take the view that serious infections occur only in the presence of severe pre-existing disease (Orie et al., 1960). It is our opinion that the invasive or necrotizing type of infection may be a more frequent cause 
of serious pulmonary disease than has previously been realized, as we have seen four separate instances in a single hospital group within a period of less than one year.

The nature of the invasive process in pulmonary tissue is controversial. It has been widely held that pre-existing tissue damage is necessary for fungal colonization to take place (Hinson et al., 1952; Bech, 1961). Primary aspergillosis in humans is regarded as a rare phenomenon, if it occurs at all. It will always be difficult to confirm that an infection is primary, when it may have taken root in some minute pulmonary lesion such as an infarct (Conen, Walker, Turner, and Field, 1962; Symmers, 1962). Once an infection is established, however, changes occurring in adjacent lung tissue are probably due to diffusible products of fungal metabolism, producing tissue reactions which may be inflammatory, granulomatous, necrotic or suppurative and which may promote further invasion by fungus (Riddell, 1958; Bech, 1961). This view arises from the observations of Henrici (1939), who extracted from the cell sap of $A$. fumigatus mycelium a thermolabile endotoxin with a pronounced histotoxic effect in laboratory animals. Clayton has also demonstrated the proteolytic activity of $\boldsymbol{A}$. fumigatus endotoxin (Campbell and Clayton, 1964). Protease systems comparable to trypsin in proteolytic and fibrinolytic activity have been isolated from centain other aspergillus species (Crewther and Lennox, 1950; Stefanini, Marin, Soardi, and Mossa, 1962). There may still be considerable variation between species, or even between different strains of a single species, in the production of such enzymes (Stefanini and Marin, 1958). Despite this, such observations lend credence to the view that an active growth of $A$. fumigatus in the lung may lead directly to pulmonary necrosis and further tissue infiltration. Even if the fungus elaborates no true exotoxin, degenerating fungal elements may liberate toxic intracellular substances which damage pulmonary tissue (Gowing and Hamlin, 1960). Susceptibility to invasive aspergillosis appears to be enhanced by antibiotics and by corticosteroids, by radiotherapy, by cytotoxic drugs, and by a number of systemic and debilitating conditions (Bech, 1961). Indeed, Sidransky and Friedman (1959) showed experimentally that the administration of antibiotics and corticosteroids to laboratory animals exposed to airborne spores of $\boldsymbol{A}$. flavus greatly increased the numbers that became infected. Each of our four patients had been given multiple antibiotic therapy, and this may have been a factor which contributed to the invasive nature of the fungal infection. In addition, it is probable that infection with the fungus was secondary to bacterial infection or other lung disease. In patient 1, Ps. aeruginosa was isolated both from the sputum and from lung tissue at necropsy. This organism itself can produce tissue necrosis, and it may be that the bacterial infection in this patient was largely to blame for the destruction of lung tissue (Pierce, Edmonson, McGee, Ketchersid, Loudon, and Sanford, 1966). The fungus would then be regarded as a secondary invader, fulfilling a saprophytic role, although still actively growing within the lungs. Okudaira and Schwarz (1962), who have observed actual invasion of living bronchial cartilage by hyphae, concede that at least a part of the cellular reaction accompanying the necrosis in lesions where aspergillus is present may be due to concomitant bacterial infection.

A major difficulty in the diagnosis of pulmonary aspergillosis is the fact that aspergillus species are common laboratory contaminants. The airborne spores of these fungi are present in the atmosphere throughout the year and the demonstration of aspergillus in sputum is not necessarily an indication of pathogenicity. A diagnosis of aspergillary infection should be based on repeated culture, on clinical, radiological, and immunological evidence, and on histological material where possible (Sarkany, 1968). In the present series of four patients, histological confirmation was available in only one case. The three others, however, all developed precipitating antibodies against $A$. fumigatus, and all yielded numerous colonies of the fungus from successive samples of sputum. Such sputum yields may be designated 'fungus abundant' and be regarded as evidence of fungal growth in the lungs rather than simple contamination (English and Henderson, 1967).

The finding of a mat of tangled hyphae lining parts of the abscess cavities in the lungs of the first patient has similarities to the necropsy appearances in a case described by Toigo (1960). One of Bech's patients, too, had abscess cavities containing sloughs of ramifying septate mycelia (Bech, 1961). A feature of the present case was the manner in which Pseudomonas bacilli and Aspergillus mycelium appeared to co-exist, large numbers of bacilli lying among the masses of hyphae. The possibility of primary invasion by the Gramnegative bacillus, with $A$. fumigatus playing a saprophytic role, has been discussed; the question of symbiosis between bacterium and fungus must also be considered. Fungal hyphae, particularly in the medium of soil, are known to present surfaces 
frequently colonized by bacteria (Warcup, 1965). The reasons for these associations are not well understood, but may range from the action of bacterial fimbriae on the hyphal surface (Duguid, Smith, Dempster, and Edmunds, 1955) to the exudation of nutrients from fungi or their substrates (Tribe, 1957). Conversely, Mangan (1969) has noted that spore germination of $A$. terreus in broth cultures is inhibited by $P$ s. aeruginosa, probably through the elaboration of pyocyanine and fluorescent green pigments which have a fungistatic effect. The factors which determined the fungalbacterial association in the present case therefore remain obscure. Another open question is the association between bronchial neoplasia and fungal infection, as observed in patient 4 . Here again it may be that an abnormal or devitalized tissue surface is available for fungal colonization. We consider that a true lung abscess, in which $A$. fumigatus was actively growing, preceded the appearance of neoplasia in the opposite lung; but the time interval was probably too short to suggest that the fungus itself may have had a carcinogenetic effect.

In ill patients, especially those with abnormal lungs and those who have received multiple antibiotic or corticosteroid therapy, it seems likely that fungal infection will be recognized more often in the future, if borne in mind and specifically looked for. Specialized mycological techniques may be necessary to establish the diagnosis. Once diagnosed, the fungal infection, if progressive, should be treated actively whether it is regarded as primary or secondary in nature. Three of the patients described were treated with natamycin, a tetraene antifungal antibiotic isolated from Streptomyces natalensis, and related to other polyenes including nystatin and amphotericin (Struyk, Hoette, Drost, Waisvisz, van Eek, and Hoogerheide, 1958). It is poorly absorbed from the gut but is effective and non-toxic when administered as an aerosol (Edwards and $\mathrm{La}$ Touche, 1964). Whether inhalation therapy can really control a deep-seated invasive infection must remain questionable. Vedder and Schorr (1969) have treated a $3 \frac{1}{2}$-year-old girl suffering from primary disseminated aspergillosis with metastatic infection of the skin by inhalations of nystatin aerosol; this treatment produced measurable blood levels of nystatin, and within a few weeks there was a satisfactory clinical recovery, although the authors are guarded about the child's ultimate prognosis. The synthetic antimycotic clotrimazole, developed in Germany, is said to be absorbed well from the alimentary tract, with few side-effects, and to have activity against almost all pathogenic fungi (British Medical Journal, 1969). It is possible that this drug may have an application in bronchopulmonary aspergillosis.

We wish to thank Dr. I. W. B. Grant, Dr. M. B. Matthews, and Professor J. A. Strong for allowing us to study their patients. We are indebted to Dr. J. C. Gould, Director, Central Microbiological Laboratories, Western General Hospital, Edinburgh, for the serological studies, and to Dr. J. M. Drennan, Consultant Pathologist, Western General Hospital, for the necropsy examination. The illustrations were provided by the Medical Photography Unit of the University of Edinburgh.

The mycological studies were undertaken as part of a research programme supported by a grant from the Scottish Hospitals Endowments Research Trust.

\section{REFERENCES}

Bech, A. O. (1961). Diffuse bronchopulmonary aspergillosis. Thorax, 16, 144.

British Medical Journal (1969). A new synthetic antimycotic (leading article). Brit, med. J., 4, 444.

Campbell, M. J., and Clayton, Y. M. (1964) Bronchopulmonary aspergillosis. Amer. Rev. resp. Dis., 89, 186.

Conen, P. E., Walker, G. R., Turner, J. A., and Field, P. (1962). Invasive primary aspergillosis of the lung with cerebral metastasis and complete recovery. Dis. Chest, 42, 88 .

Crewther, W. G., and Lennox, F. G. (1950). Preparation of crystals containing protease from Aspergillus oryzae. Nature (Lond.) 165,680 .

Duguid, J. P., Smith, I. W. Dempster, G., and Edmunds, P. N. (1955). Non-flagellar filamentous appendages ("fimbriae") and haemagglutinating activity in Bacterium coli. J. Path. Bact. 70, 335.

Edwards, G., and La Touche, C. J. P. (1964). The treatment of bronchopulmonary mycoses with a new antibiotic-pimaricin. Lancet, 1, 1349.

English, M. P., and Henderson, A. H. (1967). Significance and interpretation of laboratory tests in pulmonary aspergillosis. J. clin. Path., 20, 832.

Gowing, N. F. C., and Hamlin, I. M. E. (1960). Tissue reactions to Aspergillus in cases of Hodgkin's disease and leukaemia. J. clin. Path., 13, 396.

Grocott, R. G. (1955). A stain for fungi in tissue sections and smears. Amer. J. clin. Path., 25, 975.

Henrici, A. T. (1939). An endotoxin from Aspergillus fumigatus. J. Immunol., 36, 319.

Hinson, K. F. W., Moon, A. J., and Plummer, N. S. (1952). Bronchopulmonary aspergillosis. Thorax, 7,317 .

Longbottom, J. L., and Pepys, J. (1964). Pulmonary aspergillosis: diagnostic and immunological significance of antigens and C-substance in Aspergillus fumigatus. J. Path. Bact., 88, 141.

Mangan, A. (1969). Interactions between some aural Aspergillus species and bacteria. J. gen. Microbiol., 58, 261.

Okudaira, M., and Schwarz, J. (1962). Tracheobronchopulmonary mycoses caused by opportunistic fungi, with particular reference to aspergillosis. Lab. Invest., 11, 1053.

Orie, N. G. M., de Vries, G. A., and Kikstra, A. (1960). Growth of Aspergillus in the human lung. Amer. Rev. resp. Dis., 82, 649.

Ouchterlony, ठ̈. (1953). Antigen-antibody reactions in gels. IV. Types of reactions in co-ordinated systems of diffusion. Acta path. microbiol. scand., 32, 230.

Pierce, A. K., Edmonson, E. B., McGee, G., Ketchersid, J., Loudon, R. G., and Sanford, J. P. (1966). An analysis of factors predisposing to gram-negative bacillary necrotizing pneumonia. Amer. Rev. resp. Dis., 94, 309.

Riddell, R. W. (1958). The role of fungi as human pathogens. Proc. roy. Soc. Med., 51, 491.

Sarkany, I. (1968). Systemic mycoses. In Recent Advances in Medicine, ed. Baron, D. N., Compston, N., and Dawson, A. M., 15th ed., pp. 243-273. Churchill, London. 
Sidransky, H., and Friedman, L. (1959). The effect of cortisone and antibiotic agents on experimental pulmonary aspergillosis. Amer. J. Path., 35, 169.

Stefanini, M., and Marin, H. M. (1958). Fibrinolysis I. Fibrinolytic activity of extracts from non-pathogenic fungi. Proc. Soc. exp. Biol., 99, 504.

- Soardi, F., and Mossa, A. (1962). Fibrinolysis IX. The comparative activity in vivo of trypsin and aspergillin $O$ (mold fibrinolysin). Angiology, 13, 254.

Struyk, A. P., Hoette, I., Drost, G., Waisvisz, J. M., van Eek, T. and Hoogerheide, J. C. (1958). Pimaricin, a new antifungal
antibiotic. Antibiotics Annual, 1957-58, p. 878. Medical Encyclopedia, New York.

Symmers, W. St. C. (1962). Histopathologic aspects of the pathogenesis of some opportunistic fungal infections, as exemplified in the pathology of aspergillosis and the phycomycetoses. Lab. Invest., 11, 1073.
Toigo, A. (1960). Pulmonary aspergillosis. Amer. Rev. resp. Dis., $81,392$.

Tribe, H. T. (1957). Ecology of micro-organisms in soils as observed during their development upon buried cellulose film. Microbial Ecology, edited by R. E. O. Williams and C. C. Spicer, University Press, Cambridge. Symp. Soc. gen. Microbiol., 7, 287.

Vedder, J. S., and Schorr, W. F. (1969). Primary disseminated pulmonary aspergillosis with metastatic skin nodules. J. Amer. med. Ass., 209, 1191.

Warcup, J. H. (1965). In Ecology of Soil-borne Plant Pathogens: An International Symposium on Factors determining the Behay or of Plant Pathogens in Soil, University of California, 1963 Edited by K. E. Baker, W. C. Snyder, and others, p. 59. John Murray, London. 